Bull. Austral. Math. Soc.

$54 \mathrm{H} 15,16 \mathrm{~A} 76$

VOL. 37 (1988) $[277-291]$

\title{
NEAR-RING-SEMIGROUPS OF CONTINUOUS SELFMAPS
}

\author{
K.D. Magill, JR.
}

\begin{abstract}
We find necessary and sufficient conditions on a topological space $X$ so that $S(X)$, the semigroup of all continuous selfmaps of $X$, is isomorphic to the multiplicative semigroup of a near-ring. The analogous problem is also considered for the semigroup of all continuous selfmaps which fix some point of $X$.
\end{abstract}

\section{INTRODUCTION}

For general information about near-rings and, in particular, for terms not defined here, we suggest that [7] be consulted. In [5] Jones and Ligh introduced the notion of an NR-semigroup (abbreviation for near-ring-semigroup). A semigroup is defined to be an NR-semigroup if it is isomorphic to the multiplicative semigroup of some near-ring. The definition of a near-ring does not require its additive group to be abelian. When it is, the near-ring is referred to as an abelian near-ring. In keeping with the preceding terminology, we define a semigroup to be an abelian-near-ring-semigroup (hereafter referred to as an $A N R$-semigroup) if it is isomorphic to the multiplicative semigroup of an abelian near-ring.

Our purpose in this paper is to characterise those topological spaces $X$ such that $S(X)$ (the semigroup of all continuous selfmaps of $X$ ) is an NR-semigroup or an ANR-semigroup. It is, of course, quite well-known that if $X$ supports a group structure compatible with its topology, then one can define pointwise addition of functions $(f+g)(x)=f(x)+g(x)$ for all $f, g \in S(X)$ and $x \in X$ and the result is a near-ring with $S(X)$ as its multiplicative semigroup. The question which must be answered is, "Are there instances where there is no group structure compatible with the topology of $X$, and yet it is still possible to define a binary operation on $S(X)$ so that the result is a near-ring whose multiplicative semigroup is $S(X)$ ?" We will see that within a large class of spaces, the answer is no. In other words, if such a binary operation on $S(X)$ exists, then there is a binary operation on $X$ such that $X$, with this operation, is a topological group. Moreover, the binary operation on $S(X)$ induced by the binary operation on $X$ will coincide with the original binary operation on $S(X)$. Once we get this result, we are then in a position to make use of all those results concerning topological structure of a space which supports a topological group. We are able to

Received 10 June 1987

Copyright Clearance Centre, Inc. Serial-fee code: 0004-9729/88 \$A2.00+0.00. 
show, for example, that if $X$ is a locally compact, connected, locally connected, separable metric space with the property that every point has a neighbourhood which is an absolute retract, then $S(X)$ is an ANR-semigroup if and only if $X$ is homeomorphic to either some Euclidean $N$-space, an $M$-dimensional torus (where $M$ is either finite or countably infinite) or the product of two such spaces. We get an analogous result for 0-dimensional spaces. Specifically, we show that if $X$ is any locally compact 0-dimensional metric space, then $S(X)$ is an ANR-semigroup if and only if $X$ is either discrete, homeomorphic to the Cantor discontinuum, or homeomorphic to the product of a discrete space and the Cantor discontinuum.

The semigroup $S(X)$ does not have a zero point except in the trivial case where $X$ consists of one point. The most natural subsemigroups of $S(X)$ which do have zeros are those of the form $S_{p}(X)$ consisting of all functions in $S(X)$ which fix some point $p \in X$. The constant function which maps everything into the point $p$ is, of course, the zero of $S_{p}(X)$. We also investigate these semigroups and we show that if $X$ is a locally compact, 0 -dimensional metric space whose derived set does not consist of exactly one point and $p$ is any point of $X$, then $S_{p}(X)$ is an ANR-semigroup if and only if $X$ is either discrete, homeomorphic to the Cantor discontinuum or homeomorphic to the product of a discrete space and the Cantor discontinuum.

\section{SOME TOPOLOGICAL PRELIMINARIES}

Definition 2.1. A topological space $X$ is admissible if it is Hausdorff, first countable and for each pair of convergent sequences $\left\{x_{n}\right\}_{n=1}^{\infty}$ and $\left\{y_{n}\right\}_{n=1}^{\infty}$ where the $x_{n}$ are all distinct, and in addition, all differ from $\lim x_{n}$, there exists a positive integer $N$ and a continuous selfmap $f$ of $X$ such that $f\left(x_{n}\right)=y_{n}$ for all $n>N$.

Proposition 2.2. Every 0 -dimensional metric space is admissible.

Proof: Let $\left\{x_{n}\right\}_{n=1}^{\infty}$ and $\left\{y_{n}\right\}_{n=1}^{\infty}$ be two sequences as in the previous definition and suppose $\lim x_{n}=p$ and $\lim y_{n}=q$. Define a function $f$ by $f\left(x_{n}\right)=y_{n}$ and $f(p)=q$. Then $f$ is a continuous function from the closed subset $\left\{x_{n}\right\}_{n=1}^{\infty} \cup\{p\}$ of $X$ into $X$ and, as such, has an extension to a continuous selfmap of $X$ by Corollary 3 of $[\boldsymbol{6}, \mathrm{p} .281]$.

Proposition 2.3. Let $X$ be a first countable, normal, Hausdorff space with the property that each point of $X$ has a neighbourhood which is an absolute retract. Then $X$ is admissible.

Proof: Let $\left\{x_{n}\right\}_{n=1}^{\infty}$ and $\left\{y_{n}\right\}_{n=1}^{\infty}$ be two sequences as in Definition 2.1 with $\lim x_{n}=p$ and $\lim y_{n}=q$. Then $q$ is contained in the interior of some $H \subseteq X$ which is an absolute retract. Choose a positive interger $N$ such that $y_{n} \in H$ for $n>N$ and define $f\left(x_{n}\right)=y_{n}$ for $n>N$ and $f(p)=q$. Then $f$ is a continuous function from 
the closed subset $\left\{x_{n}\right\}_{n=N+1}^{\infty} \cup\{p\}$ of the normal space $X$ into the absolute retract $H$ and, consequently, has an extension to a continuous function which maps all of $X$ into $H$.

Some remarks. Between them, Propositions 2.2 and 2.3 assure us of an ample supply of admissible spaces. To be sure, the two classes of spaces described are just about disjoint. The only 0-dimensional spaces which satisfy the hypothesis of Proposition 2.3 are the discrete spaces. But many other spaces (not necessarily connected) also satisfy the hypothesis. Absolute retracts include all Euclidean $N$-cells, all Euclidean $N$-spaces and all dendrites $[1$, p.138] so all these spaces are admissible as well as all normal Hausdorff spaces with the property that each point has a neighbourhood which is homeomorphic to one of these spaces. In particular, all locally Euclidean spaces are admissible.

For an example of a space which is not admissible, take $X=[0,1] \times N^{\infty}$ where $N^{\infty}$ is the one-point compactification of the natural numbers. Consider

$$
\begin{aligned}
& x_{n}=(0,(n+1) / 2) \text { for } n \text { odd, } \\
& x_{n}=(2 / n,(n+2) / 2) \text { for } n \text { even, } \\
& y_{n}=x_{n+1} \text { for all } n .
\end{aligned}
$$

The sequences $\left\{x_{n}\right\}_{n=1}^{\infty}$ and $\left\{y_{n}\right\}_{n=1}^{\infty}$ both converge to $(0, \infty)$ but no matter how large one takes $N$ to be, there does not exist a continuous selfmap $f$ of $X$ with the property that $f\left(x_{n}\right)=y_{n}$ (or $f\left(y_{n}\right)=x_{n}$ ) for all $n>N$. This example together with Propositions 2.2 and 2.3 shows that the product of two admissible spaces need not be admissible.

\section{The FUll SEMIGRoup of CONTINUOUS SELFMAPS}

The first result of this section is the fundamental lemma which, among other things, tells us that for admissible spaces, $S(X)$ can be the multiplicative semigroup of a nearring only if $X$ is a topological group.

LEMMA 3.1. Let $X$ be any admissible space and let $\oplus$ be any binary operation whatsoever on $S(X)$ with the property that

$$
(f \oplus g) \circ h=(f \circ h) \oplus(g \circ h) \quad \text { for all } f, g, h \in S(x) .
$$

Fix any $a \in X$ and define a binary operation + on $X$ by

$$
x+y=(<x>\oplus<y>)(a)
$$


where $\langle x\rangle$ and $\langle y\rangle$ denote the constant functions which map everything into $x$ and $y$ respectively. Then the following statements are all valid:

$$
(f \oplus g)(x)=f(x)+g(x) \text { for all } x \in X
$$$$
\text { and } f, g \in S(X) \text {; }
$$

+ is a continuous map from $X \times X$ to $X$;

+ is associative if and only if $\oplus$ is associative;

+ is commutative if and only if $\oplus$ is commutative;

$(X,+)$ has an identity if and only if $(S(X), \oplus)$ has an identity;

$(X,+)$ is a topological group if and only if $(S(X), \oplus)$ is a group;

$(X,+)$ is an abelian topological group if and only if $(S(X), \oplus)$ is an abelian group.

Proof: Let $x \in X$ and $f, g \in S(X)$, and use both (3.1.1) and (3.1.2) to get

$$
\begin{aligned}
f(x)+g(x) & =(<f(x)>\oplus<g(x)>)(a) \\
& =((f \circ<x>) \oplus(g \circ<x>))(a) \\
& =((f \oplus g) \circ<x>)(a)=(f \oplus g)(<x>(a))=(f \oplus g)(x)
\end{aligned}
$$

which verifies (3.1.3). To show that + is continuous, suppose $\lim \left(x_{n}, y_{n}\right)=(p, q)$. Then $\lim x_{n}=p$ and $\lim y_{n}=q$. If both $p$ and $q$ are isolated, it is immediate that $\lim \left(x_{n}+y_{n}\right)=p+q$ so we assume that one of them, say $p$, is not isolated. Consequently, there is no loss of generality if we assume that the $x_{n}$ are all distinct and different form $p$. Since $X$ is admissible, there exists a positive integer $N$ and an $f \in S(X)$ such that $f\left(x_{n}\right)=y_{n}$ for all $n>N$ and it certainly follows that $f(p)=q$. Let $\delta$ be the function in $S(X)$ which is defined by $\delta(x)=x$ for all $x \in X$. Then $\delta \oplus f$ belongs to $S(X)$ and we use (3.1.3) to get

$$
\begin{aligned}
\lim \left(x_{n}+y_{n}\right) & =\lim \left(\delta\left(x_{n}\right)+f\left(x_{n}\right)\right)=\lim (\delta \oplus f)\left(x_{n}\right) \\
& =(\delta \oplus f)(p)=\delta(p)+f(p)=p+q
\end{aligned}
$$

and since $X$ (and hence $X \times X$ as well) is first countable, this proves that + is continuous.

It is immediate from (3.1.3) that if + is associative then $\oplus$ must also be associative and the converse follows just about as easily from (3.1.2) so we omit the details. Consequently, (3.1.5) is valid. 
Statement (3.1.6) follows immediately from (3.1.2) and (3.1.3) so we next consider (3.1.7). Suppose $(S(X), \oplus)$ has and identity and denote it by $e$. Choose any $b \in X$ and use (3.1.3) to get

$$
e(b)+x=e(b)+\langle x>(b)=(e \oplus<x>)(b)=<x>(b)=x .
$$

In a similar manner, $x+e(b)=x$ so that $e(b)$ is the identity for $(X,+)$. Conversely, one easily shows that if 0 is the identity for $(X,+)$, then $\langle 0\rangle$ is the identity for $(S(X), \oplus)$.

We next verify (3.1.8). Suppose $(S(X), \oplus)$ is a group. We must show that $(X,+)$ is a topological group. Again, we let $\delta$ be the map in $S(X)$ which is defined by $\delta(x)=x$ for all $x \in X$ and we let $e$ denote the identity of $(S(X), \oplus)$. Then there exists a continuous selfmap $\sigma$ of $X$ such that $\delta \oplus \sigma=\sigma \oplus \delta=e$. As we showed in the verification of (3.1.7), e(b) is the identity for $(X,+)$, regardless of which point $x \in X$ we choose. In other words, $e$ is a constant function and we will denote the point into which $e$ maps all of $X$ by 0 . With this notation, we now have $\delta \oplus \sigma=\sigma \oplus \delta=\langle 0\rangle$. This, together with (3.1.3) results in

$$
x+\sigma(x)=\sigma(x)+x=0
$$

for each $x \in X$. Thus, $\sigma(x)$ is the inverse of $x$ in $(X,+)$ and since $\sigma$ is continuous, it follows that $(X,+)$ is a topological group. Conversely, suppose $(X,+)$ is a topological group. Then $\oplus$ is associative by (3.1.5) and $(S(X), \oplus)$ has an identity by (3.1.7). Moreover, that identity is $\langle 0\rangle$ where 0 is the identity of $(X,+)$. Since $(X,+)$ is a topological group, the function $\sigma$ which sends $x$ to its inverse is continuous and consequently, $\sigma \circ f \in S(X)$ for each $f \in S(X)$. It follows from (3.1.3) that

$$
f \oplus(\sigma \circ f)=(\sigma \circ f) \oplus f=<0>
$$

so that each element in $(S(X), \oplus)$ has an inverse. Of course, (3.1.9) follows immediately from (3.1.6) and (3.1.8) and the proof is complete.

Our next two results follow from the previous lemma and they permit us to use the wealth of information available about topological groups in our investigation of NR-semigroups and ANR-semigroups. We will assume without further mention that our topological groups are all $T_{0}$. This, of course, means that they are all regular and Hausdorff [4, p19, Theorem 4.8].

Theonem 3.2. Let $X$ be an admissible space. Then $S(X)$ is an NR-semigroup if and only if there exists a topological group $G$ such that $X$ and $G$ are homeomorphic as topological spaces. 
Theorem 3.3. Let $X$ be an admissible space. Then $S(X)$ is an ANR-semigroup if and only if there exists an abelian topological group $G$ such that $X$ and $G$ are homeomorphic as topological spaces.

The proofs of the two theorems are quite similar, so we discuss only the proof of the latter.

Proof of Theorem 3.3: Suppose first that $S(X)$ is an ANR-semigroup. Then there exists an abelian near-ring $(N, \star, \cdot)$ and an isomorphism $\varphi$ from $S(X)$ onto the multiplicative semigroup of $(N, \star, \cdot)$. Define a binary operation $\oplus$ on $S(X)$ by

$$
(f \oplus g)=\varphi^{-1}(\varphi(f) \star \varphi(g)) .
$$

It is a routine (although somewhat tedious) matter to show $(S(X), \oplus)$ is an abelian group and $(f \oplus g) \circ h=(f \circ h) \oplus(g \circ h)$ for all $f, g \in S(X)$. In other words, $(S(X), \oplus, \circ)$ is an abelian near-ring. It now follows from Lemma 3.1 that there exists a binary operation + on $X$ such that $(X,+)$ is an abelian topoligical group. Thus there exists an abelian topological group $G$ such that $X$ and $G$ are homeomorphic as topological spaces.

Conversely, suppose such a topological group $G$ exists and let $t$ be any homeomorphism form $X$ onto $G$. Define a binary operation + on $X$ by

$$
x+y=t^{-1}(t(x) \star t(y))
$$

where $\star$ denotes the group operation on $G$. One shows in a straightforward manner that $(X,+)$ is an abelian topological group (which is, of course, topologically isomorphic to $(G, \star)$ ) and so now we can define a binary operation $\oplus$ on $S(X)$ by

$$
(f \oplus g)(x)=f(x)+g(x)
$$

for all $x \in X$ and $f, g \in S(X)$. Then $(S(X), \oplus, \circ)$ is an abelian near-ring which implies that $S(X)$ is an ANR-semigroup.

The next several results follow quickly from Theorems 3.2 and 3.3 and classical results from the theory of topological groups. In order to discuss these, it is convenient to indroduce some notation and to agree upon certain conventions. The symbol $\mathbf{R}^{N}$ will denote the Euclidean $N$-space and $S^{N}$ will denote the Euclidean $N$-sphere. That is

$$
S^{N}=\left\{x \in \mathbf{R}^{N+1}:\|x\|=1\right\} .
$$

$S^{1}$ is, of course, just the unit circle in $R^{2}$ and is an abelian topological group when its points are regarded as complex numbers and multiplied accordingly. The $M$ dimensional torus $T^{M}$ is the topological group formed by taking the product of $M$ 
copies of the topological group $S^{1} . T^{1}$, of course, coincides with $S^{1}$. Here, we allow $M$ to be countably infinite as well as finite so that an $M$-dimensional torus may well be the product of a countably infinite number of copies of $S^{1}$. However, when we write $\mathrm{R}^{N}$ or $S^{N}, N$ will always denote a positive integer. Each $\mathbf{R}^{N}$ is also an abelian topological group with the usual pointwise addition. There will be times when we will be regarding $\mathrm{R}^{N}$ and $T^{M}$ as topological groups and other times when we will be regarding them as topological spaces only. The context will make the situation clear. And now we are ready to prove

Theorem 3.4. $S\left(S^{N}\right)$ is an NR-semigroup if and only if $N=1$ or 3 and it is an ANR-semigroup if and only if $N=1$.

Proof: Since $S^{1}$ can be regarded as an abelian topological group, it follows immediately from Theorem 3.3 that $S\left(S^{1}\right)$ is an ANR-semigroup. Now let $G$ be the collection of all quaternions of norm 1. Under the usual multiplication of quaternions, $G$ is a nonabelian topological group whose underlying space is homeomorphic to $S^{\mathbf{3}}[\mathbf{3}$, p374]. Thus, Theorem 3.2 implies that $S\left(S^{3}\right)$ is an NR-semigroup. If $N \neq 1$ or 3 then there exists no group structure on $S^{N}$ compatible with its topology [2, p.105] so that Theorem 3.2 tells us that $S\left(S^{N}\right)$ is not an NR-semigroup when $n \neq 1$ or 3 . We have now shown that $S\left(S^{N}\right)$ is an NR-semigroup if and only if $n=1$ or 3 and $S\left(S^{1}\right)$ is an ANR-semigroup. To complete the proof we must show that, although $S\left(S^{3}\right)$ is an NR-semigroup, it is not an ANR-semigroup and because of Theorem 3.3, this will be accomplished when we are able to demonstrate that there exists no abelian group structure on $S^{3}$ which is compatible with the topology on $S^{3}$. But Theorem 42 of [8, p.169] tells us that the only compact, connected and locally connected metric spaces which have abelian group structures compatible with their topologies are the $M$-dimensional tori. Since $S^{3}$ is homeomorphic to none of these, the conclusion follows.

TheOREM 3.5. Let $X$ be a locally compact, connected, locally connected, separable metric space which is admissible. Then $S(X)$ is an ANR-semigroup if and only if $X$ is homeomorphic to either some Euclidean $N$-space, some $M$-dimensional torus or the product of two such spaces.

Proof: Suppose $X$ is homeomorphic to one of the spaces mentioned. They can all be regarded as abelian topological groups so it follows from Theorem 3.3 that $S(X)$ is an ANR-semigroup.

Conversely, suppose $S(X)$ is an ANR-semigroup, Then $X$ is homeomorphic to an abelian topological group $G$ by Theorem 3.3 and it follows from Theorem 43 of [8, p.170] that $G$ is topologically isomorphic to either some $\mathbf{R}^{N}$, some $T^{N}$ (in [8], $T$ is denoted by $K$ ) or to some $\mathbf{R}^{N} \times T^{N}$. 
Some remarks. It may appear at a glance that our hypothesis is stronger than it needs to be in order to invoke Theorem 43 of [8] but there is a blanket assumption there that the groups are second countable $[8, \mathrm{p} .126]$.

TheOREM 3.6. Let $X$ be a locally compact 0 -dimensional metric space. Then the following statements are equivalent:

$$
\begin{aligned}
& S(X) \text { is an ANR-semigroup; } \\
& S(X) \text { is an NR-semigroup; } \\
& X \text { is either discrete, homeomorphic to the Cantor } \\
& \text { discontinuum or homeomorphic to the product } \\
& \quad \text { of a discrete space with the Cantor discontinuum. }
\end{aligned}
$$

Proof: We first show that (3.6.3) implies (3.6.1). Suppose $X$ is descrete. If $X$ is finite and has $N$ elements then $X$ is homeomorphic to the underlying space of the discrete cyclic group of order $N$. If $X$ is infinite, it is homeomorphic to the underlying space of any free abelian group (endowed with the discrete topology) on any set whose cardinality agrees with that of $X$. Thus, Theorem 3.3 applies and we conclude that $S(X)$ is an ANR-group whenever $X$ is discrete.

Now let $\mathbb{Z}_{2}$ denote the cyclic group of order two and let $G$ be the topological group formed by taking the product of a countably infinite number of copies of $Z_{2}$. $G$ is certainly abelian and moreover, its underlying space is homeomorphic to the Cantor discontinuum [2, p24] so if $X$ is homeomorphic to the Cantor discontinuum, then $S(X)$ is an ANR-semigroup. Finally, since any discrete space $Y$ can be endowed with an abelian group structure which is compatible with its topology, then the product $Y \times G$ is also an abelian topological group so that $S(X)$ is an ANR-semigroup whenever $X$ is homeomorphic to such a space.

It is immediate that (3.6.1) implies (3.6.2) so that we have only to show that (3.6.2) implies (3.6.3). Suppose $S(X)$ is an NR-semigroup. Then Theorem (3.2) tells us that $X$ is homeomorphic to a topological group $G$. Since a topological group is homogeneous, either no point of $G$ is isolated or all points of $G$ are isolated. If all points are isolated, then $G$ (and hence $X$ as well) is discrete. We consider the case where no point of $G$ is isolated. According to Theorem (7.7) of [4, p.62], $G$ contains a compact open subgroup $H$. Topologically, $H$ is a compact 0 -dimensional metric space with no isolated points and, consequently, is homeomorphic to the Cantor discontinuum [9, p217, Corollary 30.4]. For any $a \in G$ the map $t$ defined by $t(x)=a x$ (we use multiplicative notation) is a homeomorphism from $G$ onto itself which carries $H$ onto the left coset of $a H$. Thus each left coset is an open subset of $G$ which is homeomorphic to the Cantor discontinuum and so we have $G=\cup\left\{H_{\alpha}: \alpha \in \Lambda\right\}$ where the $H_{\alpha}$ are the mutually 
disjoint left cosets. For each $\alpha \in \Lambda$, let $h_{\alpha}$ be a homeomorphism from $H_{\alpha}$ onto the Cantor discontinuum $K$ and define a map from $G$ onto $K \times \Lambda$ (let $\Lambda$ have the discrete topology) by $h(x)=\left(h_{\alpha}(x), \alpha\right)$ where $H_{\alpha}$ is the coset which contains $x$. Then $h$ is a homeomorphism and we see that in this case, $X$ is homeomorphic to the product of a discrete space with the Cantor discontinuum. We remark that if $\Lambda$ is finite, then $K \times \Lambda$ is homeomorphic to $K$. This is not the case if $\Lambda$ is infinite since then $K \times A$ is not compact.

We conclude this section with one more result.

THEOREM 3.7. Let $X$ be a countable metric space. Then the following statements are equivalent:

$$
\begin{aligned}
& S(X) \text { is an } A N R \text {-semigroup; } \\
& S(X) \text { is an NR-semigroup; } \\
& X \text { is homeomorphic to either the countably infinite } \\
& \quad \text { discrete space or to the space of rational numbers }
\end{aligned}
$$

Proof: (3.7.3) implies (3.7.1) by Theorem (3.3) so all we really need to show is that (3.7.2) implies (3.7.3). Suppose (3.7.2) holds and let $G$ be a group which is homeomorphic to $X$. Since any toplogical group is homogeneous, $G$ is either discrete or no point is isolated. But a countably metric space with no isolated points is homeomorphic to the space of rational numbers by a well-known result of Sierpinski $[6$, p.287] and the conclusion follows.

\section{Semigroups OF THE FORM $S_{p}(X)$}

Recall that $S_{p}(X)$ denotes the semigroup of all continuous selfmaps of $X$ which fix the point $p \in X$. As we mentioned in the introduction, these are perhaps the most natural subsemigroups of $S(X)$ which contain zeros. We characterise in this section certain 0-dimensional spaces, metric spaces $X$ for which $S_{p}(X)$ is an NR-semigroup or an ANR-semigroup. In contrast to the situation for $S(X)$, any near-ring having $S_{p}(X)$ for its multiplicative semigroup will have a two-sided multiplicative zero. Our first two results are straightforward consequences of Theorems 3.2 and 3.3 .

TheOREM 4.1. Let $X$ be any admissible space and let $p$ be any point of $X$. If $S(X)$ is an NR-semigroup then so is $S_{p}(X)$.

Theorem 4.2. Let $X$ be any admissible space and let $p$ be any point of $X$. If $S(X)$ is an ANR-semigroup then so is $S_{p}(X)$.

The proofs being similar, we discuss the proof of the former only. 
Proof of Theorem 4.1: By Theorem 3.2, there exists a topological group $G$ homeomorphic to $X$. Since $G$ is homogeneous, there exists a homomorphism $t$ from $X$ onto $G$ such that $t(p)=0$, the identity of $G$. Let $\star$ denote the group operation on $G$ and define a binary operation + on $X$ by

$$
x+y=t^{-1}(t(x) \star t(y)) \text {. }
$$

One can verify that $(X,+)$ is a topological group whose identity is $p$. Now define a binary operation $\oplus$ on $S_{p}(X)$ by

$$
(f \oplus g)(x)=f(x)+g(x)
$$

for all $x \in X$ and $f, g \in S_{p}(X)$. Then $\left(S_{p}(X), \oplus, 0\right)$ is a near-ring and the proof is complete.

The converses to Theorems 4.2 and 4.3 probably hold, at least for an extensive class of spaces, if not for the class of admissible spaces. At this point, we are able to prove the converses only when our spaces are certain 0-dimensional metric spaces. We first prove an analogue of Lemma 3.1 for $S_{p}(X)$ and it is convenient to have some additional notation for this. Let $x$ be any point in $X$ distinct from $p$ and let $G$ be a clopen subset of $X$ which contains $x$ but not $p$. The symbol $\langle x, G\rangle$ will denote the function which is defined by $\langle x, G\rangle(y)=x$ for all $y \in G$ and $\langle x, G\rangle(y)=p$ for $y \in X-G$. Note that $\langle x, G\rangle$ is continuous and fixes the point $p$. Consequently, $<x, G>\in S_{p}(X)$ for each $x \neq p$. We will refer to such a function as an $x$-function or an $x$-map.

Lemma 4.3. Let $X$ be a 0 -dimensional metric space, let $p$ be any point of $X$ and suppose that if $p$ is nonisolated, then $X$ contains at least one other point, distinct from $p$, which is also nonisolated. Let $\oplus$ be any binary operation whatsoever on $S_{p}(X)$ which satisfies:

$$
\begin{aligned}
(f \oplus g) \circ h & =(f \circ h) \oplus(g \circ h) \text { for all } f, g, h \in S_{p}(X), \text { and } \\
<p>\oplus f & =f \oplus<p>=f \text { for all } f \in S_{p}(X)
\end{aligned}
$$

where $\langle p\rangle$ is the constant function which maps everything into the point $p$. Now we define a binary operation + on $X$. For any $x \in X$, define

$$
p+x=x+p=x \text {. }
$$

For $x, y \neq p$, choose a clopen set $G$ containing both $x$ and $y$ but not $p$. Then choose any $a \in G$ and define

$$
x+y=(<x, G>\oplus<y, G>)(a)
$$


The definition of $x+y$ does not depend on either the clopen set $G$ or the point a. Moreover, the following statements are all valid:

$$
\begin{aligned}
& (f \oplus g)(x)=f(x)+g(x) \text { for all } x \in X \text { and } \\
& \quad f, g \in S_{p}(X) ; \\
& +\quad \text { is a continuous map from } X \times X \text { to } X ; \\
& +\quad \text { is associative if and only if } \oplus \text { is associative; } \\
& +\quad \text { is commutative if and only if } \oplus \text { is commutative; } \\
& (X,+) \text { is a topological group if and only if } \\
& \quad\left(S_{p}(X), \oplus\right) \text { is a group; } \\
& (X,+) \quad \text { is an abelian topological group if and } \\
& \quad \text { only if }\left(S_{p}(X), \oplus\right) \text { is an abelian group. }
\end{aligned}
$$

Proof: We first prove that for $x, y \neq p$, the definition of $x+y$ does not depend upon the choice of the clopen set $G$ or the point $a$. Let $\langle x, G\rangle$ and $\langle x, H\rangle$ be two $x$-maps, let $\langle y, G\rangle$ and $\langle y, H\rangle$ be the corresponding $y$-maps and let $a \in G$ and $b \in H$. We then use (4.3.1) several times and we get

$$
\begin{aligned}
(< & x, G>\oplus<y, G>)(a)=((<x, G>\oplus<y, G>) \circ<a, G \cup H>)(a) \\
& =((<x, G>0<a, G \cup H>) \oplus(<y, G>0<a, G \cup H>))(b) \\
& =(<x, G \cup H>\oplus<y, G \cup H>)(b) \\
& =((<x, H>0<b, G \cup H>) \oplus(<y, H>0<b, G \cup H>))(b) \\
& =((<x, H>\oplus<y, H>) \circ<b, G \cup H>)(b)=(<x, H>\oplus<y, H>)(b) .
\end{aligned}
$$

Next, we verify (4.3.5) and we consider several cases.

Case 1. $x=p$.

Here, we must have $(f \oplus g)(p)=f(p)+g(p)$ for all $f, g \in S_{p}(x)$. But this is an immediate consequence of (4.3.3) and the fact that each function in $S_{p}(X)$ fixes the point $p$.

Case 2. $x \neq p$ and $f(x)=p$.

In what follows, we take any $x$-map $\langle x, G\rangle$ and we use both (4.3.2) and (4.3.3).

$$
\begin{aligned}
(f \oplus g)(x) & =((f \oplus g) \circ<x, G>)(x)=((f \circ<x, G>) \oplus(g \circ<x, G>))(x) \\
& =(<p>\oplus(g \circ<x, G>))(x)=(g \circ<x, G>)(x)=g(x) \\
& =p+g(x)=f(x)+g(x) .
\end{aligned}
$$

The case where $x \neq p$ and $g(x)=p$ follows in much the same way, and so we are left with: 
Case 3. $x \neq p, f(x) \neq p$ and $g(x) \neq p$.

Let $G$ be any clopen set containing $x, f(x)$ and $g(x)$ but not $p$ and choose any $a \in G$. We use both (4.3.1) and (4.3.4) and we get

$$
\begin{aligned}
(f \oplus g)(x) & =((f \oplus g) \circ<x, G>)(a)=((f \circ<x, G>) \oplus(g \circ<x, G>))(a) \\
& =(<f(x), G>\oplus<g(x), G>)(a)=f(x)+g(x) .
\end{aligned}
$$

This completes the verification of (4.3.5) and we turn to (4.3.6). If $X$ is discrete, it is immediate that + is continuous on $X \times X$ so we consider the case where $X$ is not discrete and one of our assumptions assures us of the existence of a nonisolated point $z \neq p$. Thus there exists a sequence $\left\{z_{n}\right\}_{n=1}^{\infty}$ of points distinct from each other and from $p$ such that $\lim z_{n}=z$. Now suppose $\lim \left(x_{n}, y_{n}\right)=(x, y)$. Then $\lim x_{n}=x$ and $\lim y_{n}=y$. Define two functions $f$ and $g$ by $f\left(z_{n}\right)=x_{n}, f(z)=x, f(p)=p$, $g\left(z_{n}\right)=y_{n}, g(z)=y$ and $g(p)=p$ Then $f$ and $g$ are continuous functions from the closed set $\{z, p\} \cup\left\{z_{n}\right\}_{n=1}^{\infty}$ into the 0 -dimensional metric space $X$ and, as such, can be extended to continuous selfmaps of $X$ [6, p.281, Corollary 3]. We will denote the respective extensions by $f$ and $g$ as well. Evidently, both $f$ and $g$ belong to $S_{p}(X)$ and we use (4.3.5) to get

$$
\begin{aligned}
\lim \left(x_{n}+y_{n}\right) & =\lim \left(f\left(z_{n}\right)+g\left(z_{n}\right)\right)=\lim (f \oplus g)\left(z_{n}\right) \\
& =(f \oplus g)(z)=f(z)+g(z)=x+y .
\end{aligned}
$$

Thus, + is a continuous binary operation on $X$. We will give an example later to show that + need not be continuous if $p$ is the only nonisolated point of $X$.

It is immediate from (4.3.5) that $\oplus$ is associative if + is associative. Conversely, suppose $\oplus$ is associative and consider points $x, y, z \in X$. If any of these points is equal to $p$ then it is immediate from (4.3.3) that $(x+y)+z=x+(y+z)$ so assume $x, y, z \neq p$ and choose a clopen set $G$ containing $x, y$ and $z$ but not $p$ and let $a$ be any point in $G$. We then have

$$
\begin{aligned}
(x+y)+z & =(<x, G>\oplus<y, G>)(a)+<z, G>(a) \\
& =((<x, G>\oplus<y, G>) \oplus<z, G>)(a) \\
& =(<x, G>\oplus(<y, G>\oplus<z, G>))(a) \\
& =<x, G>(a)+(<y, G>\oplus<z, G>)(a)=x+(y+z) .
\end{aligned}
$$

Statement (4.3.8) is an immediate consequence of (4.3.5) and we now consider (4.3.9). If $(X,+)$ is a topological group, it follows form (4.3.5) that $\left(S_{p}(X), \oplus\right)$ must be a group. Conversely, suppose $\left(S_{p}(X), \oplus\right)$ is a group. According to (4.3.2) and (4.3.3) respectively, $\langle p\rangle$ is the identity of $\left(S_{p}(X), \oplus\right)$ and $p$ is the identity of $(X,+)$. Recall 
that $\delta$ denotes the map in $S_{p}(X)$ which is defined by $\delta(x)=x$ for all $x \in X$. Then there exists a map $\sigma \in S_{p}(X)$ such that $\delta \oplus \sigma=\sigma \oplus \delta=<p>$. From (4.3.5), we get

$$
x+\sigma(x)=\delta(x)+\sigma(x)=(\delta \oplus \sigma)(x)=<p>(x)=p .
$$

Similarly, $\sigma(x)+x=p$ so that $\sigma(x)$ is the inverse of $x$ in $(X,+)$. Thus, $(X,+)$ is a group. Moreover, + is continuous by (4.3.6) and since $\sigma$ is also continuous, we conclude that $(X,+)$ is, in fact, a topological group. Statement (4.3.10) now follows from (4.3.8) and (4.3.9) and the proof of the lemma is finally complete.

An example. We remarked after verifying (4.3.6) that + need not be continuous if $p$ is the only nonisolated point of $X$. To see this, let $X=\{0\} \cup\{1 / n\}_{n=1}^{\infty}$ have the topology induced by the real line and consider $S_{0}(X)$. Define a map $t$ from $X$ onto the non-negative integers by $t(0)=0$ and $t(1 / n)=n$. Then for $f, g \in S_{0}(X)$ and $x \in X$, define

$$
(f \oplus g)(x)=t^{-1}(t(f(x)) \star t(g(x)))
$$

where here, $\star$ denotes the usual addition of integers (the symbol + will be used to denote the binary operation on $X$ induced by $\oplus)$. Certainly, $(f \oplus g)(0)=0$ and one shows that $f \oplus g$ is continuous on $X$. Thus, $f \oplus g \in S_{0}(X)$ so that $\oplus$ is a binary operation on $S_{0}(X)$. Moreover, one can show that both (4.3.1) and (4.3.2) are satisfied when $p=0$. However, the binary operation + defined by (4.3.3) and (4.3.4) is not continuous in this case. To see this, note that $\lim (1 / n, 1)=(0,1)$ and

$$
\begin{aligned}
1 / n+1 & =(<1 / n,\{1 / n, 1\}>\oplus<1,\{1 / n, 1\}>)(1) \\
& =t^{-1}(t \circ<1 / n,\{1 / n, 1\}>(1) \star t \circ<1,\{1 / n, 1\}>(1)) \\
& =t^{-1}(t(1 / n) \star t(1))=t^{-1}(n+1)=1 /(n+1) .
\end{aligned}
$$

Thus $\lim (1 / n+1)=0$ and not 1 as it would need to be in order for + to be continuous. $\left(S_{0}(X), \oplus\right)$ is a commutative semigroup with identity $\langle 0\rangle$. However, it is far from being a group in that no element other than $\langle 0\rangle$ has an inverse. Consequently, $\left(S_{0}(X), \oplus, 0\right)$ is not a near-ring.

And now we prove two results which, among other things, give us the converses to Theorems 3.2 and 3.3 for the spaces of Lemma 3.4.

Theorem 4.5. Let $X$ be a 0 -dimensional metric space, let $p \in X$ and suppose that if $p$ is nonisolated, then some other point in $X$, distinct form $p$, is also nonisolated. Then the following statements are equivalent:

$$
S(X) \quad \text { is an NR-semigroup; }
$$$$
S_{p}(X) \text { is an NR-semigroup; }
$$

there exists a topological group $G$ such that $X$ and $G$ are homeomorphic as topological spaces. 
Theorem 4.6. Let $X$ be a 0 -dimensional metric space, and let $p \in X$ and suppose that if $p$ is nonisolated, then some other point in $X$, distinct form $p$, is also nonisolated. Then the folowing statements are equivalent:

$$
\begin{aligned}
& S(X) \quad \text { is an ANR-semigroup; } \\
& S_{p}(X) \text { is an ANR-semigroup; } \\
& \text { there exists an abelian topological group } G \text { such that } \\
& \quad X \text { and } G \text { are homeomorphic as topological spaces. }
\end{aligned}
$$

The proofs being similar, we discuss only the proof of Theorem 4.5.

Proof of TheOREM 4.5: It follows immediately from Theorem 3.2 that (4.5.1) and (4.5.3) are equivalent and Theorem 4.1 assures us that (4.5.1) implies (4.5.2). The proof will be complete when we are able to show that (4.5.2) implies (4.5.3) and a technique used previously works here as well. Let $\varphi$ be an isomorphism from $S_{p}(X)$ onto the multiplicative semigroup of some near-ring $(N, \star,+)$. Define a binary operation $\oplus$ on $S_{p}(X)$ by

$$
f \oplus G=\varphi^{-1}(\varphi(f) \star \varphi(g)) .
$$

Since $\langle p\rangle$ is the two-sided zero of $S_{p}(X)$, it follows that $\varphi(<p>)$ is the additive identity of the near-ring $(N, \star, \cdot)$. One shows in a straightforward manner that $\left(S_{p}(X), \oplus\right)$ is a group and that both (4.3.1) and (4.3.2) are satisfied. We now take + to be the binary operation on $X$ defined by (4.3.3) and (4.3.4) and it follows from (4.3.9) that $(X,+)$ is a topological group. This concludes the proof.

Theorem 4.7. Let $X$ be a locally compact 0 -dimensional metric space, let $p$ be any point of $X$ and suppose that if $p$ is nonisolated, then some other point in $X$, distinct from $p$ is also nonisolated. Then the following statements are all equivalent:

$$
\begin{array}{ll}
\text { (4.7.1) } & S(X) \quad \text { is an ANR-semigroup; } \\
(4.7 .2) & S(X) \quad \text { is an NR-semigroup; } \\
(4.7 .3) & S_{p}(X) \text { is an ANR-semigroup; } \\
(4.7 .4) & S_{p}(X) \quad \text { is an NR-semigroup; } \\
(4.7 .5) & X \quad \text { is either discrete, homeomorphic to the Cantor } \\
& \text { discontinuum or homeomorphic to the product of a } \\
\text { discrete space with the Cantor discontinuum. }
\end{array}
$$$$
\text { (4.7.4) } \quad S_{p}(X) \text { is an NR-semigroup; }
$$

Proof: The result follows from Theorems 3.6, 4.5 and 4.6.

In a similar manner, Theorems $3.7,4.5$. and 4.6 immediately yield: 
Theorem 4.8. Let $X$ be a countable metric space, let $p \in X$ and suppose that if $p$ is nonisolated, then $X$ contains some other point, distinct from $p$, which is also nonisolated. Then the following statements are all equivalent:

$$
\begin{aligned}
& S(X) \quad \text { is an ANR-semigroup; } \\
& S(X) \text { is an NR-semigroup; } \\
& S_{p}(X) \text { is an ANR-semigroup; } \\
& S_{p}(X) \text { is an NR-semigroup; } \\
& X \quad \text { is homeomorphic to either the countably infinite } \\
& \quad \text { discrete space or to the space of rational numbers. }
\end{aligned}
$$

In conclusion, we remark that one obvious open problem is to get converses to Theorems (4.1) and (4.2) for spaces other than the ones we have discussed in this section. In particular, we conjecture that it can be done for admissible, locally compact, connected, locally connected, separable metric spaces. It would then follow from Theorem (3.5) that for any such space $X$ and any $p \in X, S_{p}(X)$ would be an ANR-semigroup if and only if $X$ was homeomorphic to either some Euclidean $N$-space, some $M$-dimensional torus or to a product of two such spaces.

\section{REFERENCES}

[1] K. Borsuk, 'Theory of retracts', in Polska Akademia Nauk Monograffie Matamatyczne (Polish Sci. Pub, Warszawa, 1967).

[2] N. Bourbaki, General topology Part 2, Hermann, Paris, Addison-Wesley, Reading Mass., 1966.

[3] N. Bourbaki, Lie groups and Lie algebras, Part 1, Hermann, Paris, Addison-Wesley, Reading, Mass., 1975.

[4] E. Hewitt and K.A. Ross, Abstract harmonic analysis I (Springer-Verlag, New York, 1979).

[5] P. Jones and S. Ligh, 'Finite near-ring semigroups', Pacific J. Math. (88) 2 (1980), 491-504.

[6] K. Kuratowski, Topology, Vol. I (Academic Press, New York, 1966).

[7] G. Pilz, Near-rings, North-Holland Math. Studies 23, New York, 1977.

[8] L. Pontrjagin, Topological groups (Princton University Press, 1939).

[ө] S. Willard, General Topology (Addison-Wesley, Reading, Mass., 1970).

\footnotetext{
Department of Mathematics

SUNY at Buffalo

Buffalo, New York 14214-3093

United States of America
} 